\title{
Intelligent Robotics Navigation System: Problems, Methods, and Algorithm
}

\author{
Siti Nurmaini ${ }^{1}$, Bambang Tutuko ${ }^{2}$ \\ ${ }^{1,2}$ Robotic and Control Research Lab. Faculty of Computer Science, Universitas Sriwijaya, Indonesia
}

\begin{tabular}{|c|c|}
\hline Article Info & ABSTRACT \\
\hline Article history: & This paper set out to supplement new studies with a brief and \\
\hline Received Jul 20, 2017 & $\begin{array}{l}\text { comprehensible review of the advanced development in the area of the } \\
\text { navigation system, starting from a single robot, multi-robot, and swarm }\end{array}$ \\
\hline Revised Sep 14, 2017 & robots from a particular perspective by taking insights from these biological \\
\hline Accepted Sep 30, 2017 & $\begin{array}{l}\text { systems. The inspiration is taken from nature by observing the human and the } \\
\text { social animal that is believed to be very beneficial for this purpose. The }\end{array}$ \\
\hline Keyword: & $\begin{array}{l}\text { intelligent navigation system is developed based on an individual } \\
\text { characteristic or a social animal biological structure. The discussion of this }\end{array}$ \\
\hline Navigation & paper will focus on how simple agent's structure utilizes flexible and \\
\hline Intelligent & $\begin{array}{l}\text { potential outcomes in order to navigate in a productive and unorganized } \\
\text { surrounding. The combination of the navigation system and biologically }\end{array}$ \\
\hline Problems & inspired approach has attracted considerable attention, which makes it an \\
\hline Methods & important research area in the intelligent robotic system. Overall, this paper \\
\hline Algorithms & $\begin{array}{l}\text { explores the implementation, which is resulted from the simulation } \\
\text { performed by the embodiment of robots operating in real environments. }\end{array}$ \\
\hline
\end{tabular}

Copyright @ 2017 Institute of Advanced Engineering and Science. All rights reserved.

\section{Corresponding Author:}

Siti Nurmaini,

Robotic and Control Research Lab., Faculty of Computer Science,

Universitas Sriwijaya,

Jl. Raya Palembang-Prabumulih, KM. 32 Indralaya Ogan Ilir, Palembang, Indonesia.

Email: sitinurmaini@gmail.com

\section{INTRODUCTION}

One of the most significant and vital issues in the study of mobile robot revolves around the navigation system due to the need of clearly pinpointing it in the design phase. It is important to note that several issues have been solved which include perception, cognition, action, human-robot interaction, and control system [1]-[5]. In most cases, the problems can be easily overcome when a mobile robot is restricted to a finite domain, for example, a house, factory, and office. A number of capabilities which include control ability, obstacle avoiding, trajectory planning, and safe distance to the goal are needed by a mobile robot in order to produce excellent navigation. Each navigation system must address the aforementioned common designs to ensure that all tasks can be accomplished. The conventional control systems have proposed a number of approaches to solve the existing challenges, which involve using a rigid model but with several constraints [6]-[9]. However, the mobile robot interactions among sensor, actuator, and its environment are known to be problematic to express in mathematical models [10]-[14]. Therefore, it is believed to restrict the relevance of such control system design in this application [15],[16]. In addition, the conventional control approaches tend to disintegrate mobile robot behaviors into a sense model-plan-act type [17]. Subsequently, it is discovered to produce complex equations for both environmental mapping and hard computation. Apart from that, the model is only appropriate for a certain type of environmental situation. Therefore, uncertainty and imprecision of the surrounding may cause the mobile robot to be stacked at a local minimum or stopped at one point. Moreover, the complexity of mobile robot behavior becomes greater when a number of tasks are added to the control system for the purpose of achieving the target [18]-[20]. 
The navigation system is constructed based on learning techniques that provide the competence to reason under environmental uncertainty as well as to observe from different exposure, which is very necessary to ensure that the robot can be controlled and able to produce a good performance. However, the design will be difficult to build as a result of several factors such as inherent uncertainties in the unorganized surrounding, incomplete perceptual information, and imprecise actuators. In light of this, the navigation system design should be able to do the followings: (i) react effectively to unpredictable situations immediately after they happen, (ii) consider multiple concurrent requirements in the process, and (iii) reach the target based on a specification object. Most of the established works mainly considered navigation tasks only for single robot. Meanwhile, a great growing interest has been observed in cooperative approaches, which makes communication as one of the vital focus [21]-[23]. In some environmental situations, the navigation functions are extremely difficult to be overcome using a single robot. The large area for sensing tends to be the reason that causes the environmental condition to pose a post-disaster relief and target searching in military applications. Hence, a single robot must be designed with powerful structures and hardware equipment to ensure all the functions are in order [24]. In this case, a more expensive design cost, computational resources, and larger memory are highly required to overcome this issue. However, it is important to note that if the robot fails, the whole system may be affected.

Currently, the simplest form of a group of robots based on a network developed by many researchers [25]-[28]. The robotics system is made to function based on a cooperative approach in order to communicate with each other for the purpose of conducting tasks that are difficult to be performed on their own. Generally, this particular joint system is known as swarms robots which are made to function based on their biological counterparts and with system solution that is highly dependent on three characteristics, namely self-organization, self-adaptiveness, and emergence [29]-[31]. The characteristics are based on the fact that the swarm's organization originates "from within the system not imposed from outside or it comes from local interactions between individual robot in a decentralized way" [29],[30]. In some cases, they are required to move between two places whereby the collective navigation has made it possible to function [22],[29]-[33]. The swarm formation must be controlled due to the facts that all robots work in a particular group with one target. The swarm formation control is performed without a designated leader; hence, the control and communication system are highly desirable. On top of that, the swarm robots are simple hardware, which explains the limited computational cost. All requirements are very vital parameters in the design of the robot. However, it needs to be known that this is very difficult to compute in reality and may not be relevance to all possible surroundings. Therefore, these challenges must be overcome by developing simple and robust algorithms for the purpose of controlling and coordinating these very large groups of robots. The overall structure of this paper takes the form of five sections described as follows: Section 2 offers a brief overview of the mobile robot navigation issue. Section 3 is concerned with the concise methods used by the navigation system. Section 4 presents a review on single robot compare to swarm robots navigation algorithm. Finally, Section 5 provides a concise summary of the entire findings of this study.

\section{MOBILE ROBOT NAVIGATION PROBLEMS}

The type of robot in the study of navigation can be divided into three systems, namely single robot system, multi-robots system, and swarm robots system. Generally, the differences between multi-robot and swarm robots rely on the form and task of a particular system. Multi-robot is designated as a small number of robots, which have different shapes and functions that are able to work together to achieve the same goal. On the other hand, swarm robots are described as a substantial amount of simple robots that have similar shape and function. Most of the time, they work together using a local communication and coordination in order to accomplish the tasks. In this case, a navigation system built in all mobile robots has allowed them to exploit the sensing, processing, and actuating capabilities in making a control decision. In this system, it is required for the mobile robot to find a route with less risk of colliding in order to travel from a starting point to another until the target destination is reached, which remains static in the case of single robot navigation. However, the obstacles in multi-robot and swarm robots are implemented to be static and dynamic in order to account for the case of the robots moving together in an unfamiliar surrounding to find the target. This further suggests that the robot can be a dynamic obstacle. Therefore, the implementation of all single robot, multi-robots, and swarm robots should be capable of moving in a real-time trajectory with many difficulties arising from the surrounding to arrive at the specific target. However, several considerations related to several issues concerning the implementation, uncertainties, imprecision, and incomplete information in realworld unorganized surrounding.

In the navigation process, the perception and cognition are the crucial tasks in acquiring knowledge about the environment as well as how to execute the control commands performed through several sensors and actuators. The navigational system of a mobile robot can be divided into four types based on the 
interaction between the perception in the sensors process and control process in the actuators described as follows: map-based navigation, behavior-based navigation, learning based navigation, and communicationbased navigation. All the navigational tasks, behaviors, and types of the robot used in the navigation system presented in Figure 1 are further described in this section.

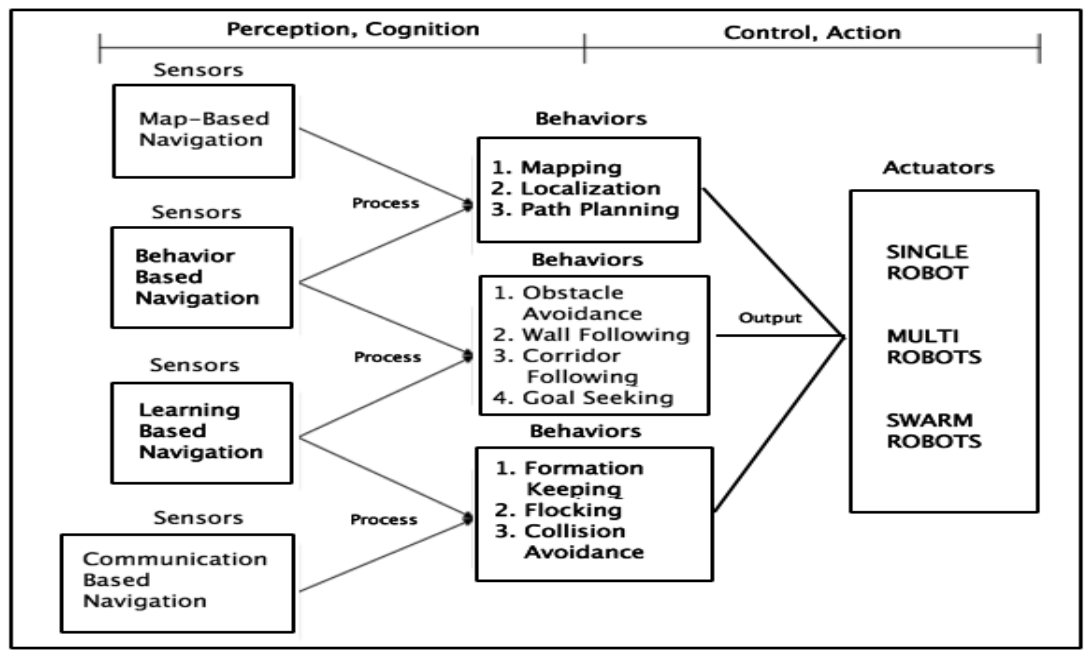

Figure 1. Navigation system based on perception-actuation

\subsection{Mapping-based Navigation}

The navigation system with environmental mapping is possible to be described as a combination of three paramount competencies as follows: mapping, localization, and path planning [34]-[38]. Environmental mapping is built in the mobile robot using a memorizing approach to allow it to fully move and explore the environment. In addition, the localization process is used for determining the present spot of the robot within the environmental mapping. The mean of deciding a particular movement to reach the goal using the map and localization process is known as a path planning process. All competencies tend to provide the robot with the capability to figure out its current location within its reference structure, which then leads to the planning of a path to reach a certain goal locations. On top of that, the navigation models based on this approach must compromise between maps and estimated position of the robot. It is very crucial for the process to ensure that the robot is able to control its approximate location given any situation and surrounding. However, there are some drawbacks to such approach, which include the fact that the approach relies only on the local sensing and environment. Hence, it is very necessary for it to also be equipped with powerful sensors or combinations. Finally, the algorithm must ensure that the uncertainties for all sensors element are able to produce imprecision and unpredictability when operating [15],[39].

\subsection{Behavior-based Navigation}

There is a total of four competencies in behavior-based navigation: obstacle avoidance, wall following, corridor following, and target seeking. Behavior induced by numerous simultaneous goals is possible to be smoothly blended into a dynamic sequence of control action. Moreover, the navigation system design is expected to express acceptable behavioral traits that were set as the possible control action. Apart from that, it can cause possible conflict in the movement of the mobile robot, particularly when it works in an actual unorganized environment. Behavior-based navigation can be developed by combining two processes such as environmental mapping and robot behaviors. Specifically, the map represents environmental situation, while the robot move is utilized by its behavior. In another situation, the system should be applied in two conditions if only one of the two processes is used, which highly depends on the implementation and interaction with other concurrent robot behaviors. However, two major problems are bound to occur when this particular approach is employed: (i) the combination of two simple behaviors in forming a complex one, and (ii) the integration of more than two behaviors.

\subsection{Learning-based Navigation}

The conventional methods make it necessary for the robot to be designed in a powerful manner with the inclusion of several sensors, actuators, and controller without having to consider the troubles that may be 
caused by the surrounding. Hence, several methods can be adopted by the navigation system in overcoming these challenges and issues. Previously published studies have proposed artificial intelligent methods to solve navigation problem, which is related to the process conducted based on learning ability. However, several key concerns must be taken into account in order to include artificial intelligent in mobile robot navigation, which is incomplete problems, imprecision, inaccurate, and uncertainty condition when they interact with possible surroundings. This particular system is only compatible with single robot behaviors and multi robot behaviors. However, a certain situation such as complex environment makes it very difficult for a robot to manage all tasks, thus producing more errors in a control process. Moreover, if some components in the system and the function of the robot failed to perform well; hence, it shows that the fault tolerance characteristic is not supported in large-scale environment. Therefore, considerably more research of intelligence navigation on mobile robot will need to be done in discovering new methods that can help to overcome the existing challenges.

\subsection{Communication-based Navigation}

A robotic system based communication is a new platform in the area of intelligence navigation on the mobile robot. In the case of navigation, the process is associated with the arrangement of huge amount of plain physical robots, which is conducted through local communication modification and sensing. In the world of robotic system, they are particularly known as swarm robots. Their operation requires a number of methods with some characteristic, which include simple autonomous platform, decentralized control, and several works on some sense of biological inspiration, and the importance of cooperation and coordination [30],[33],[40]-[42]. More specifically, swarm robots are unique because they communicate with each other instead of relying on the use of maps [43], map-building strategies [36], and external infrastructure [44]. However, the problems regarding the conflicting constraints of swarm robots are very hard to overcome, particularly concerning the situation whereby a dynamic surrounding requires an optimal path to be routed in actual-time and when a new restriction occurs. Apart from that, this problem arises due to the need of swarm robots to maneuver towards their target location while also trying to comply and adjust to their paths in considering for any possible incidence with other robots and static obstacles. Moreover, the presence of many robots and real-time constraints has caused the robots to compute their motions independently and in a decentralized manner. In this case, those animals that possess behavioral program are found to be flexible enough to adapt to the encountered environmental changes such as insect colonies [45],[46], flocks of birds [45],[47], school of fish [45],[48], and groups of amoeba [49]. The algorithm that is built based on the simple behavioral rule is for two purposes: (i) ability to minimize the need for complexity in the informationprocessing system, and (ii) ability to allow the production of behavior to optimize energetic expenditures.

The entire nature of the system is caused by individual interaction with one another. Natural selection tends to favor optimization principles that utilize simple rules as well as inherent flexibility without the need to explicitly select certain features. Therefore, the navigation output will be robust, thus making it possible to deal with erroneous circumstances, which include sensors and actuators noise as well as the ability of fault tolerance characteristic. However, the optimization that is based on the nature of animal social approach possess a few disadvantages such as: (i) inability to control the robots motion, (ii) only able to be optimal locally, (iii) produce sloppy global movements when more than one robot maneuver in a complex environment, and (iv) the possibility of the robots to be a trap in a local minimum [50]-[52]. Therefore, there is an urgent need to address the existing challenges that are important to the design requirement which include actual-time, unorganized, and dynamic surrounding as well as the problems of imprecision, incomplete, and uncertainty in a single robot and swarm robots navigation system. On top of that, failed communication and imperfect algorithm are treated as particular concerns during the development of the navigation system. Finally, the performance of the entire robotic navigation systems can either be improved or at least not degraded if all the parameters have been analyzed.

\section{METHODS OF NAVIGATION SYSTEM}

In this section, the navigation systems are reviewed in depth for the purpose of providing a number of vital information to the study of single robot being transformed to swarm robots. The comparative analysis of the three methods, namely conventional artificial intelligent, soft computing, and swarm intelligence which are related to mobile robot navigation system is presented in Figure 2. In addition, all the definitions and process related to this research will be further described in this section, including a comprehensive discussion of the findings of this study.

IJECE Vol. 7, No. 6, December 2017 : 3711 - 3726 


\subsection{Conventional Artificial Intelligence}

The most primitive navigation strategy was developed to produce robots that are able to accomplish several tasks assigned to them [53]-[55]. However, the adaptive technique must be combined with particular strategy. Moreover, the navigation only seems to work on local environment; therefore, the robot will have difficulties to recognize and control its motion if the surrounding is always changing which will consequently affect the accomplishment of the mission. In addition, a huge amount of conventional artificial intelligence (AI) approaches are necessary to overcome the existing limitation which include artificial potential field methods [56], virtual target approach [57], landmark learning [58], tangent graph [59], path velocity decomposition method [60], accessibility graph [61], space-time concept [62], incremental planning [63], relative velocity approach [64], reactive control scheme [17], curvature-velocity method [65], dynamic window approach [9], and Simultaneous Localization and Mapping (SLAM).

Unfortunately, the mentioned conventional AI approaches seem to undergo the following disadvantages: (i) local controller [66], (ii) high computational resources that are caused by a large number of state [67],[68], (iii) absence of optimization module [69], (iv) regular dead-lock situation due to local minimum [70], (v) absence of passage between closely spaced obstacles which results in oscillations [56]. Therefore, it is highly recommended for the control strategy to be developed in order to produce an acceptable solution to mobile robot navigation problems.

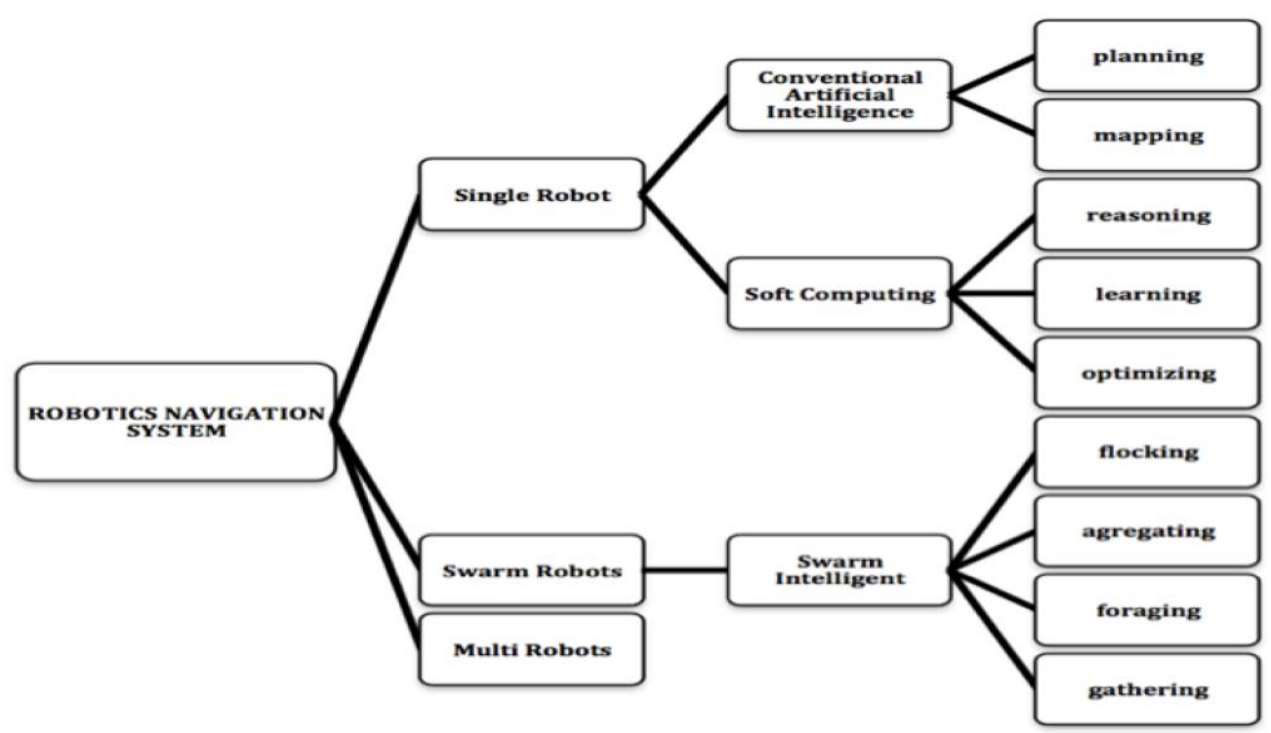

Figure 2. Intelligent robotics navigation system algorithms

\subsection{Soft Computing}

The purpose of Soft Computing (SC) methods is to achieve a robust and low-cost solution. Hence, this method has proposed a methodology that utilizes a number of knowledge in reference to the remarkable ability of the human mind to reason and learn [71]. On top of that, it also provides an alternative solution to clarify some of the aforementioned navigation problems. This particular method is different from the conventional AI methods because it does not produce imprecision, uncertainty, partial truth, and approximation. This technique has been extensively utilized in the design of mobile robot application and has resulted in good performance [72]-[75]. The ability to deal with unorganized and unfamiliar environments has made this a suitable technique to address robotic control issues as well as navigation problem. These techniques are believed to bring effective methods and improve the intelligence in mobile robot navigation. A few type of the soft computing techniques include fuzzy logic system, neural network, and genetic algorithm.

The fuzzy logic system is an excellent solution for mobile robot navigation due to the systems' inherent imprecision, especially type-1 fuzzy logic system (T1FLS). However, T1FLS is incapable of fully handling the uncertainties [76] as a result of the restricted modeling of T1FLS membership functions (Mfs) in minimizing the effect of uncertainty. Meanwhile, the uncertainty value will disapear when MFs can at least be given partially [77]. In this case, the error will still occur and a small error is still significant because it has the ability to negatively affect the navigation performance [78]. Recently, a new kind of fuzzy logic known type-2 fuzzy logic system (T2FLS) has been established as the improved version of T1FLS and proven to be 
successful in mobile robot navigation [79]-[81]. In particular, T2FLS are extremely beneficial in a situation whereby it is difficult to conclude the actual measurement [77]. Moreover, T2FLS are computationally intensive and hard to be built for actual-time application, especially for mobile robot navigation [82],[83]. Meanwhile, interval type-2 fuzzy logic system (IT2FLS) is proposed to simplify the computation. IT2FLS possess the possibility to solve the restrictions of T1FLS as well as to produce a new generation of the fuzzy system with improved performance of the navigation system [84],[85]. However, IT2FLS is still computationally in high demand compared to T1FLS [86].

Another case of navigation research reveals a surrounding that is imprecise, vast, dynamical, and unstructured. Hence, it is very important for a mobile robot to be able to understand a particular surrounding in order to reach the target without collisions [10]. Moreover, data processing, recognition, learning, reasoning, interpreting, decision-making, and action capacities must be endowed with perception. In order to build an adaptable navigation system in a mobile robot, neural networks (NNs) possess the ability to observe the situations and emulate the remarkable perception and pattern recognition for each environment. For the past few years, previously published studies have reported an issue in reference to NNs as well as its application in order to better assist the mobile robot to produce an advanced development of their operational capabilities in an unfamiliar surrounding [87]-[89]. The process of faulty or noisy data by the NNs is more valuable compared to the classical AI techniques because NNs are known to be highly tolerant to noises [90]. On top of that, numerous studies have successfully applied the NNs technique for the purpose of developing the model related to mobile robot navigation. However, the major disadvantage of conventional NNs technique refers to the repeated presentation of training data required in actual-time, which often results in a very long learning time.

Reasoning, decision making, and learning have been proposed as part of the SC techniques; however, all results must be optimized in order to achieve an excellent performance in navigation system, especially in the effort of figuring out the optimal value of the target position. In complex optimization problem, Genetic Algorithm (GA) has been determined as one of the most strong algorithms. On top of that, GA is presented as an emerging optimization method and its fundamental properties have made GA as an attractive choice for finding a solution to the problem related to mobile robot navigation [91]-[93]. Apart from that, GA can also solve the following issues caused by the traditional search techniques which include the gradient-based methods: (1) high computational cost, (2) large memory spaces, and (3) time to consume [94]. However, the implementation of GA algorithm in mobile robot navigation finds it difficult to generate a global optimum solution as well as produce slow convergence [93].

All intelligent soft computing techniques possess different characteristics which include the ability to learn and explain the process of making the appropriate decision for a particular type of problem and not generalize it for others. In regard to this, neural networks possess a number of learning ability and excellent capability of recognizing patterns. However, neural networks are not competence in clarifying how decisions can be made [95]. On top of that, fuzzy logic systems are very good at determining their own decisions and addressing the reasons for inaccurate information and uncertainty [96]. However, they have difficulties to immediately obtain the rules that are set for the purpose of producing the best decisions. Evolutionary Algorithm (EA) generates an excellent performance in the optimization process which has been used in a great variety of applications with a high success rate. The algorithm imitates the manner evolution acts, which then allows the performance of controllers to be improved or be adapteds to different systems. However, GA is associated with random numbers that are probabilistic, locally optimum, and with slow convergence [97]. Several characteristics of soft computing technique in mobile robot application are described in Table 1. However, there are a number of restrictions related to soft computing techniques which makes it hard for navigation tasks to be performed in large-scale environment. Finally, they are unable to guarantee the robustness and fault tolerance characteristic because they are related to centralized control architecture and does not support self-organization.

Table 1. Soft computing performance in intelligent navigation

\begin{tabular}{|c|c|c|c|c|c|}
\hline Algorithm & Process & Behavior & Adaptability & Computational & Word problems \\
\hline Type-1 Fuzzy & reasoning and & perception to & low & low rate & uncertainty \\
\hline $\begin{array}{c}\text { Logic } \\
\text { Type-2 Fuzzy }\end{array}$ & $\begin{array}{l}\text { decision- making } \\
\text { reasoning and }\end{array}$ & $\begin{array}{l}\text { action } \\
\text { perception to }\end{array}$ & medium & high rate & $\begin{array}{c}\text { and imprecision environment } \\
\text { uncertainty }\end{array}$ \\
\hline $\begin{array}{l}\text { Interval Type-2 } \\
\text { Fuzzy Logic }\end{array}$ & $\begin{array}{l}\text { reasoning and } \\
\text { decision- making }\end{array}$ & $\begin{array}{l}\text { perception to } \\
\text { action }\end{array}$ & medium & medium rate & $\begin{array}{c}\text { uncertainty and imprecision } \\
\text { environment }\end{array}$ \\
\hline Neural Networks & $\begin{array}{l}\text { learning and } \\
\text { adapting }\end{array}$ & $\begin{array}{c}\text { human capabilities } \\
\text { to learn and adapt }\end{array}$ & high & high rate & $\begin{array}{l}\text { The dynamic environment } \\
\text { under varying conditions }\end{array}$ \\
\hline
\end{tabular}

IJECE Vol. 7, No. 6, December 2017: $3711-3726$ 


\subsection{Swarm Intelligence}

Soft Computing method contradicts the method of Swarm Intelligence (SI) because its algorithms are developed based on the knowledge of individuals as human beings, whereas the algorithms of SI are established based on the behaviors of social creatures such as insects and the animal that live in groups. Hence, the behavior of social insect becomes the main concept of Swarm Intelligence (SI) which can be further categorized into autonomy, distributed functioning, and self-organizing for the purpose of constructing numerous artificial systems [30]. The implementation of SI method in navigation system especially in multi-robot and swarm robots are the result of distributed functioning, a communication for autonomy, as well as the cooperation and coordination of self- organizing with all the group of robots. Therefore, the proposed methods will have to consider the aforementioned requirements. Multi-robotic and Swarm robotics system seem to share the similar properties of SI, in which the cooperative behaviors of robots activities interacting locally with their environment is analyzed. Moreover, it produces an excellent performance in the navigation system, particularly for the complex system in a predefined environment. The different types of SI methods include particle swarm optimization (PSO) [104], ant colony optimization (ACO) [105],[106], bee colony optimization (BCO) [107], and firefly algorithm (FA) [108]. Similar to other methods, each of the methods under the SI approach tends to pose a number of strengths and limitations. However, there is no best optimization technique that can be used to solve the problems. It is important to note that the set of parameters and suitable methods are responsible for defining the quality of the swarm robots navigation solution.

PSO algorithm is a population-based optimization method that was suggested by Kennedy and Eberhart in 1995. The insight of this method is extracted from the social behavior of a flock of bird and a school of fishes. In most cases, PSO is adopted in numerous optimization areas due to its exclusive searching mechanism, simple concept, computational efficiency, and easy implementation [98]. Hence, its simplicity has led to various robotics navigation problems, which are solved by utilizing PSO algorithm in order to produce good performance [24],[99]-[102]. The information is gathered from sensors on a real-time robot during the navigation process. This navigation process is comprised of three stages. First, the navigation issue is turned into an optimization problem. Next, the proper objective function is constructed in reference to the goal and obstacles. Finally, the key advantage of PSO refers to fast convergence in various complex optimizations and search challenges [103],[102]. Meanwhile, population-based heuristics are more expensive due to higher reliance upon the function values instead of the subordinate data. However, PSO is exposed to incomplete convergence, especially when it involves many possible conclusion or dimensions that can be optimized that can easily fall into local optima [109]-[111].

The Ant Colony Systems (ACS) is regarded as one of the heuristic approaches. Hence, the solution to the problem of combinatorial optimization is known as the ACS process, which was conducted in accordance with the innate nature of ants, particularly in the mechanism of cooperation and practice [112]. Meanwhile, another colony approach, which is in regard of heuristic algorithm, is known as Ant Colony Optimization (ACO). The key concept of ACO is to idealize a problem in regard to looking for the basic cost path in a graph. The ACO contradicts the ACS in the form of pheromone trails [105],[113],[114]. In the case of ACO, the pheromone is upgraded in two ways, which are locally and a global updating rule in order to adjust the pheromone level on the edges that is assigned to the finest existing ant tour. According to the literature, both ACS and ACO were discovered to generate robust and flexible skills in order to manage various optimization challenges. In addition, ACO has also been adjusted to number of odor source localization [41],[115],[116]. Other than that, it also presents two ant-inspired robot foraging algorithms, which generate a better arrangement between the robots [117]. Overall, the utilization of ACO algorithm in swarm robots applications tends to generate excellent achievement in the optimization process [31],[118],[119]. Nevertheless, the ACO algorithm in navigation system seems to possess a few disadvantages as a result of the dependent process of ACO, which results in the unclear time of convergence. Various natural systems have demonstrated that very basic individual organisms are able to form systems that can conduct extremely difficult work by dynamically communicating with one another.

The artificial bee communities are deemed to share similar behavior and are regarded to be slightly different from the natural bees. Hence, the artificial bee colony optimization (BCO) is believed to be capable of solving constricted optimization issues. Apart from that, the BCO possess the ability to settle deterministic combinatorial problems, including combinatorial problems that are categorized by uncertainty [107]. Other than that, BCO has been utilized for the purpose of devising path in mobile robots [120]-[122]. The challenges of this study refer to the effort of finding out the trajectory of motion of the robots. This process begins from a predefined starting position to a permanent target position in the world map with the final aim of reducing the route distance of all the robots. The algorithm comprises of a recruitment method to collaborate the established findings with other robots of the swarm, including a navigation plan to navigate in an unfamiliar world. The BCO algorithm is useful in generating an effective solution to overcome the issues 
of path planning, which consequently decrease the time for the path to emerge. However, the algorithm is local convergence, which is incapable of securing the global convergence because they are absolutely random [125]-[126]. Therefore, the algorithm must be improved in order to present an environment that is equipped with certainty, dynamic, and stochastic property.

Firefly algorithm (FF) is known to generate short and rhythmic flashes. Specifically, the design of flashes is generally exclusive for a particular species. Two basic roles of such flashes are to draw the attention of mating partners (communication) and potential prey [108]. In the swarm robots application, firefly is utilized in the process of devising path as well as fault tolerance characteristic [127]-[129]. The path planning has become a major challenge in the navigation of mobile robots, with the focus of figuring out the best path with the minimum risk of collision in a given surrounding. Generally, there are different routes that can assist the robot to arrive at a particular target, but it is important to note that the best path has to be chosen based on the established guideline.

Table 2. Swarm intelligence performance in intelligent navigation

\begin{tabular}{|c|c|c|c|c|}
\hline Algorithm & Process & Behavior & Computational & Word problems \\
\hline $\begin{array}{l}\text { Particle Swarm } \\
\text { Optimization }\end{array}$ & $\begin{array}{l}\text { aggregating } \\
\text { and flocking }\end{array}$ & $\begin{array}{c}\text { coordinate motion and collective } \\
\text { exploration }\end{array}$ & low rate & $\begin{array}{c}\text { target seeking, path planning, } \\
\text { localization }\end{array}$ \\
\hline $\begin{array}{l}\text { Ant Colony } \\
\text { Optimization }\end{array}$ & $\begin{array}{l}\text { foraging and } \\
\text { trailing }\end{array}$ & $\begin{array}{l}\text { collective transport, task allocation } \\
\text { and consensus achievement }\end{array}$ & medium rate & $\begin{array}{l}\text { path planning, obstacle avoidance, } \\
\text { trail avoidance, mapping }\end{array}$ \\
\hline Bee Colony & foraging & task allocation and consensus & low rate & path planning, localization \\
\hline Optimization & & achievement & & \\
\hline $\begin{array}{l}\text { Firefly } \\
\text { Algorithm }\end{array}$ & gathering & $\begin{array}{l}\text { collective fault detection and group } \\
\text { size regulation }\end{array}$ & medium rate & path planning and fault tolerance \\
\hline
\end{tabular}

The key benefits of FA are described as the automatic subdivision as well as the competency to compromise with multimodality [123]. In the case of mobile robot navigation, it generates the outcomes in finding the perfect path with the following characteristics: shortest path, least energy consuming, or shortest time. However, it is possible for the swarm robots to be trapped into several local optimums as a result of the inability of firefly algorithm to recall or learn any past events with a better situation, thus causing them to move without the recollection of its previous better situation which can result in missing conditions [124]. All the approaches that can be used to describe the comparison between swarm intelligence algorithm and application in real-world problems are summarized in Table 2.

Table 3 presents the main differences among conventional artificial intelligence, soft computing, and swarm intelligence approaches, particularly in terms of software, hardware, and algorithm requirements.

Table 3. Comparison of three approaches in navigation system

\begin{tabular}{cccc}
\hline Performance & Conventional AI & Soft Computing & Swarm Intelligence \\
\hline Processing time & slow & medium & fast \\
Computational & high & medium & low \\
Complexity & high & medium & low \\
Scalability & low & low & high \\
Adaptability & nil & low & high \\
Typical application & single agent & single agent/multi-agent & multi-agent \\
Environment & known & known/unknown & unknown \\
Algorithm design & human experience & human and animal behavior & social animal \\
Control architecture & centralized & centralized & decentralized \\
Design characteristic & powerful hardware & powerful hardware & simple hardware \\
Cost & high & high & low
\end{tabular}

\section{SINGLE ROBOT VS SWARM ROBOTS NAVIGATION ALGORITHMS}

\subsection{Single Robot}

Intensive reviews have been conducted between conventional AI and Soft Computing (SC) research in mobile robot navigation systems. Table 4 provides a summary of strengths and limitations of related approaches. On top of that, several comparisons of related technologies are displayed, especially regarding the utilization of soft computing technique in solving the inherent limitations of the navigation system. As can be observed in Table 4, a clear comparison is described between conventional AI approach and Soft Computing approach in mobile robot application. The traditional AI seem to provide full attention to the effort of imitating human intelligence through the use of symbol manipulation and symbolically organized 
knowledge bases. This particular intelligence is exhibited by machines or software. On the other hand, this approach restricts the conditions that can be applied by conventional AI. Meanwhile, the importance of mapping and planning in controlling the movement of mobile robot seems to further generate more advantageous and disadvantageous. It is vital to note that the surrounding of the mobile robot is actual-time, always changing, and unorganized. Hence, it limits the implementation as a result of requiring a precisely stated analytical model and often a huge amount of computation time.

$\mathrm{SC}$ is known to be a part of computational intelligence technique. More specifically, it refers to a group of nature-inspired computational techniques and procedures in order to focus on complicated realworld issues. SC contradicts the conventional AI in its effects as well as the role model for soft computing which is the human mind. It is highly lenient of imprecision, uncertainty, partial truth, and approximation. Therefore, these advantages are very beneficial in intelligent navigation system design due to the presence of imprecision in sensor detection, uncertainty in dynamic environment, and error in the actuator. On top of that, SC is exploited to overcome the challenges and produce high performance of the navigation system, including simple and flexible algorithm for the purpose of navigation and communication. A number of characteristics are required to be exhibited by the robots to ensure excellent functonality, namely the ability to prevent any possible crash, cover the terrain effectively, distribute the task, assisting one another with more data through various sensors, and the capability of generating an unfixed redistribution to adhere to the situation provided if the robot is unable to function [29]. Hence, it is without doubt that a great attention must be given to the process of controlling the robot teams. However, it is important to acknowledge the difficulty of the procedure due to its ability to complicate the system [130]. In addition, several types of conventional centralized method have been utilized [130],[131], but no significant limitations managed to be detected, thus it cannot treated as a general-purpose solution. The disadvantages of centralized control include high computational cost and communication complexity, lack of flexibility, and unreasonable robustness [132].

Table 4. Strengths and limitations of conventional AI and soft computing approach in navigation systems

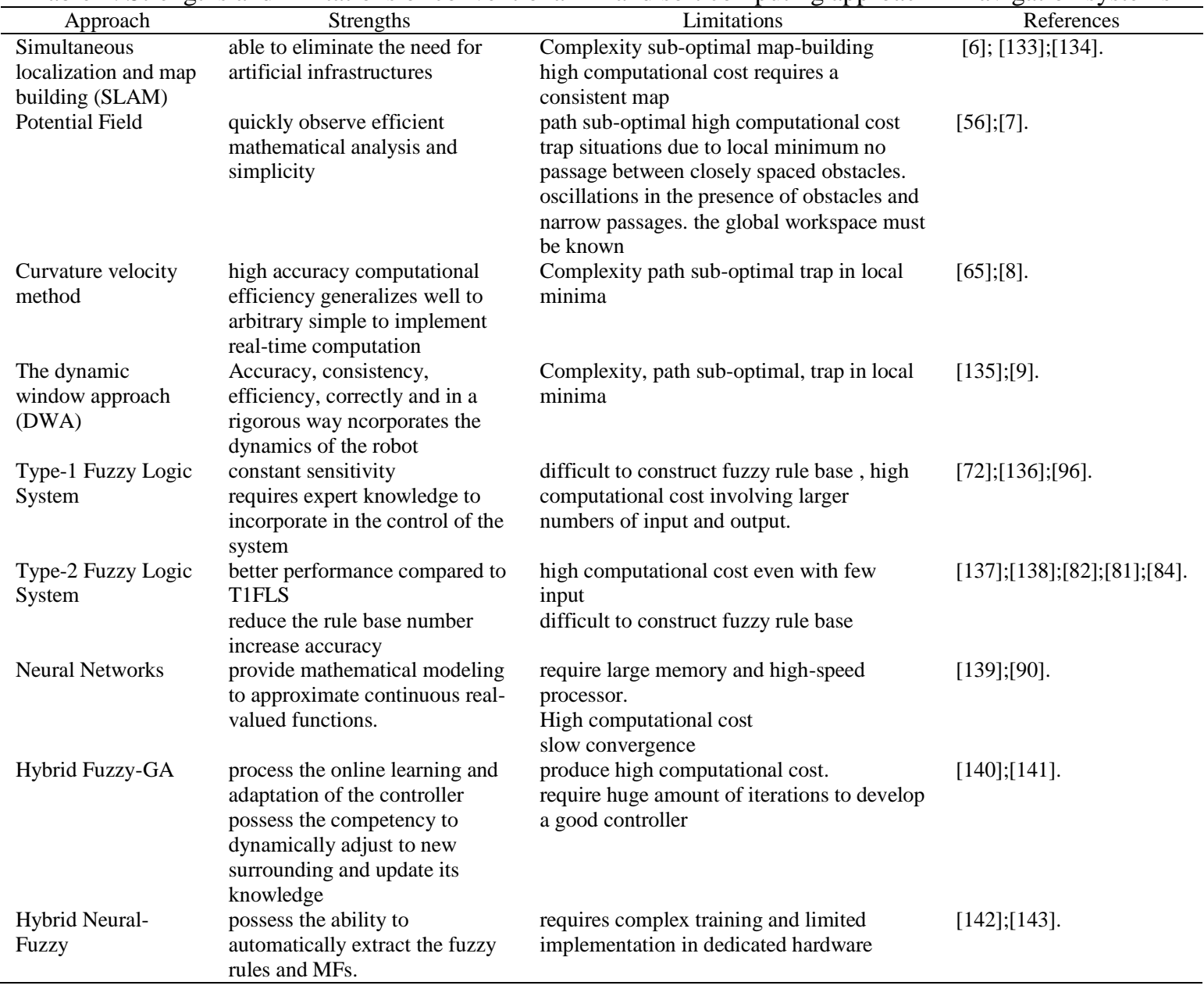




\subsection{Swarm Robots}

Swarm robots are widely known to be one of the highly crucial application areas in swarm intelligence. In this case, assigned control approach is deemed to be more appropriate for the control of systems which involve a huge amount of robots, including for the systems whereby the information about the surrounding is able to be collected or sensed by the robots themselves. Swarm robots are inspired by biological evolution, which also consequently develop behaviors from animals in order to produce optimal collective decisions concerning the navigation of individual robots as well as to generate efficient and robust navigation. In this situation, each robot tends to determine its individual action by recognizing the present surrounding they are in and employing a number of predefined control laws. The central proposal is to construct control laws that will allow the entire robot system to accomplish the target objectives such as collision-free navigation or building a spatial structure.

Currently, a number of swarm intelligence-based optimization algorithms have been suggested by numerous researchers in order to overcome the traditional centralized algorithms which include PSO, ACO, BCO, and FA. They move in a systematic manner without any coordinator. They are processed in simple code and low computational resources, whereby the individual system is believed to have the ability to transform its movement mode when the computational price is high. As for mobile robot navigation, swarm intelligence is proposed in order to figure out an optimal and collision-free route from a starting point to the target point in unfamiliar and changing surrounding [104]-[108]. The common swarm intelligence system possesses the listed fundamental principle characteristics such as proximity, quality, diverse response, stability, and adaptability. Finally, this review provides a number of comparisons on the related algorithm, especially on the utilization of the swarm intelligent algorithm in solving the inherent limitations of mobile robot navigation.

Table 5. Strengths and limitations of swarm intelligence approach in navigation system

\begin{tabular}{|c|c|c|c|}
\hline Algorithm & Strengths & Limitations & References \\
\hline Particle Swarm & easy to implement & premature convergence & [50];[144];[145]. \\
\hline \multirow[t]{5}{*}{ Optimization } & few parameter control & slow convergence & \\
\hline & low computation & optimality convergence & \\
\hline & great optimization ability fast & influenced by inertia weight & \\
\hline & convergence & low flexibility & \\
\hline & $\begin{array}{l}\text { good implementation in swarm } \\
\text { robots }\end{array}$ & trap in local minima & \\
\hline Ant Colony & distributed computation & difficult analysis & {$[104] ;[113] ;[114] ;$} \\
\hline Optimization & $\begin{array}{l}\text { dynamic application } \\
\text { good result in swarm robots search } \\
\text { and exploration }\end{array}$ & $\begin{array}{l}\text { slow convergence } \\
\text { uncertain time to converge }\end{array}$ & [105]. \\
\hline Bee Colony & Fast convergence & high computational cost & [107];[121]. \\
\hline \multirow[t]{2}{*}{ Optimization } & high flexibility & poor convergence & \\
\hline & $\begin{array}{l}\text { global optimization } \\
\text { support implementation of parallel } \\
\text { processing }\end{array}$ & local optimization & \\
\hline \multirow[t]{5}{*}{ Firefly Algorithm } & the high convergence rate & slow convergence speed & [127]; [128]. \\
\hline & low computational cost & the algorithm inflexible algorithm & \\
\hline & less number of iterations and & parameters do not change with the & \\
\hline & floating point & time & \\
\hline & suitable for parallel processing & Local optimızation & \\
\hline
\end{tabular}

Table 5 shows swarm intelligence is suitable for simple agents, but with basic behavior and consciousness. The control structure is dispersed due to the absence of global information in the system. Moreover, the collapse of an individual agent is tolerated when mobile robots move dynamically in every changing surrounding. As far as swarm robots application is concerned, it is difficult to design the navigation system concerning the parameters because they may provide a dramatic effect related to the emergence of collective behavior. On the other hand, the individual behavior appears like noise. Moreover, there is no analytical mechanism and the collective behavior of swarm robots cannot be inferred from single robot behavior in the certain situation. In addition, it is necessary for the forms of coordination employed in swarm robots to take into consideration the uncertainty, limitation, and mistakes that arise from the processing method of sensor information. Other than that, each existing algorithms for swarm robots navigation possess its own strengths and restrictions that are related to a specific goal, which also considers the importance of priority among different performance. Finally, several algorithms managed to be briefly explained from a respective point of view. 


\section{CONCLUSION}

The present study has examined the problems, methods, and application of the advanced mobile robot navigation system. This study also set out to contribute new data to the literature of this particular area of study as well as to identify areas that require further research. Mobile robot navigation is considered as one of the main application areas, which have gathered numerous attentions due to its wide potential application. Moreover, general algorithms were observed to meet development obstacle in this field, which include complex computing and high dependence on high-precision sensors. The competency to navigate in any surrounding is vital in mobile robot application to avoid any hazardous situations such as collisions and serious conditions. Therefore, it is required to keep the stability of the trajectory and formation in order to reach the target in a short time. Basically, the navigation can be achieved with three combination algorithms, namely self-localization, path planning, and map building. Hence, several conventional environment algorithms have been proposed using an environmental model. However, it is difficult to analyze sensing, actuating, and interaction with the mobile robot in a particular surrounding. Apart from that, it was discovered that the algorithm does not ensure uncertainty, impression, and inaccuracy in the dynamic environment. Hence, the intelligent algorithm is proposed to improve the performance as well as to overcome the disadvantages related to mobile robot navigation. On top of that, the computational intelligent algorithm, which includes soft computing and swarms intelligent are considered as powerful approaches that can provide the solution without modeling the environment. In regard to this, three competencies were built, namely reasoning, learning, and optimizing. Unfortunately, it is best to acknowledge that not all algorithms are suitable for the general task because each task has to follow its own specific criteria. Finally, if the algorithms are combined, a good performance in the single robot, the multi-robot, and the swarm robots system is achieved.

\section{ACKNOWLEDGEMENT}

The authors wish to express their utmost gratitude to Universitas Sriwijaya and Kementerian Riset Teknologi dan Pendidikan Tinggi Indonesia for their strong and continuous support in this research work as well as for the grant of Unggulan Perguruan Tinggi 2017 and Hibah Bersaing 2017.

\section{REFERENCES}

[1] S. Thrun and A. Bücken, "Integrating grid-based and topological maps for mobile robot navigation," in Proceedings of the National Conference on Artificial Intelligence, pp. 944-951, 1996.

[2] A. Elfes, "Using occupancy grids for mobile robot perception and navigation," Computer, vol/issue: 22(6), pp. 46$57,1989$.

[3] M. A. Goodrich and A. C. Schultz, "Human-robot interaction: a Survey," Foundations and trends in humancomputer interaction, vol/issue: 1(3), pp. 203-275, 2007.

[4] E. Gat, "Integrating planning and reacting in a heterogeneous asynchronous architecture for controlling real-world mobile robots," in $A A A i$, vol. 1992, pp. 809-815, 1992

[5] R. C. Arkin, "Integrating behavioral, perceptual, and world knowledge in reactive navigation," Robotics and autonomous systems, vol/issue: 6(1), pp. 105-122, 1990.

[6] Y. Yingmin and X. Hu, "Robot Simultaneous Localization and Mapping Based on Self-Detected Waypoint," Cybernetics and Information Technologies, vol/issue: 16(2), pp. 212-221, 2016.

[7] S. S. Ge and Y. J. Cui, "Dynamic motion planning for mobile robots using potential field method," Autonomous Robots, vol/issue: 13(3), pp. 207-222, 2002.

[8] J. L. Fernández, et al., "Improving collision avoidance for mobile robots in partially known environments: the beam curvature method," Robotics and Autonomous Systems, vol/issue: 46(4), pp. 205-219, 2004.

[9] D. Fox, et al., "The Dynamic Window Approach to Collision Avoidance," IEEE Robotics and Automation Magazine, vol. 23, pp. 23-33, 1997.

[10] A. Saffiotti, "The uses of fuzzy logic in autonomous robot navigation," Soft Computing, vol/issue: 1(4), pp. 180197, 1997.

[11] O. Motlagh, et al., "An expert fuzzy cognitive map for reactive navigation of mobile robots," Fuzzy Sets and Systems, vol. 201, pp. 105-121, 2012.

[12] M. R. Akbarzadeh-T, et al., "Soft computing for autonomous robotic systems," Computers \& Electrical Engineering, vol/issue: 26(1), pp. 5-32, 2000.

[13] K. Tanaka, et al., "Novel method of estimating surface condition for tiny mobile robot to improve locomotion performance," in Intelligent Robots and Systems. IEEE/RSJ International Conference on, pp. 6515-6520, 2015.

[14] M. Algabri, et al., "Comparative study of soft computing techniques for mobile robot navigation in an unknown environment," Computers in Human Behavior, vol. 50, pp. 42-56, 2015.

[15] S. Nurmaini, et al., "An embedded interval type-2 neuro-fuzzy controller for mobile robot navigation," in Systems, Man and Cybernetics, SMC. IEEE International Conference on., pp. 4315-4321, 2009.

[16] A. Saxena and A. Saxena, "Review of Soft Computing Techniques used in Robotics Application," International Journal of Information and Computation Technology, vol/issue: 3(3), pp. 101-106, 2013. 
[17] R. A. Brooks, “A robust layered control system for a mobile robot," Journal of Robotics and Automation, vol/issue: 2(1), pp. 14-23, 1986.

[18] L. P. Kaelbling, "Learning in embedded systems," MIT press, 1993.

[19] S. Nurmaini, "Intelligent Navigation in Unstructured Environment by using Memory-Based Reasoning in Embedded Mobile Robot," European Journal of Scientific Research, vol/issue: 72(2), pp. 228-244, 2012.

[20] U. Nehmzow, "Physically embedded genetic algorithm learning in multi-robot scenarios," The pega algorithm, pp. $115-123,2002$.

[21] R. Fierro, et al., "Cooperative control of robot formations," in Cooperative control and optimization, Springer US, pp. 73-93, 2002.

[22] F. Ducatelle, et al., "Cooperative navigation in robotic swarms," Swarm Intelligence, vol/issue: 8(1), pp. 1-33, 2014.

[23] J. V. Hook, et al., "Algorithms for cooperative active localization of static targets with mobile bearing sensors under communication constraints," Robotics, IEEE Transactions on, vol/issue: 31(4), pp. 864-876, 2015.

[24] S. Nurmaini, et al., "Pattern Recognition Approach for Swarm Robots Reactive Control with Fuzzy-Kohonen Networks and Particle Swarm Optimization Algorithm," Journal of Telecommunication, Electronic and Computer Engineering, vol/issue: 8(3), pp. 155-160, 2016.

[25] P. Corke, et al., "Localization and navigation assisted by networked cooperating sensors and robots," The International Journal of Robotics Research, vol/issue: 24(9), pp. 771-786, 2005.

[26] G. S. Sukhatme, et al., "Design and development of a wireless robotic networked aquatic microbial observing system," Environmental Engineering Science, vol/issue: 24(2), pp. 205-215, 2007.

[27] V. Kumar, et al., "Networked robots," in Springer Handbook of Robotics, Springer Berlin Heidelberg, pp. 943-958, 2008.

[28] K. Kamei, et al., "Cloud networked robotics," Network, IEEE, vol/issue: 26(3), pp. 28-34, 2012.

[29] E. Sahin, "Swarm robotics: From sources of inspiration to domains of application," in Swarm robotics, Springer Berlin Heidelberg, pp. 10-20, 2004.

[30] M. Brambilla, et al., "Swarm robotics: a review from the swarm engineering perspective," Swarm Intelligence, vol/issue: 7(1), pp. 1-41, 2013.

[31] M. Senanayake, et al., "Search and tracking algorithms for swarms of robots: A survey," Robotics and Autonomous Systems, vol. 75, pp. 422-434, 2016.

[32] M. Dorigo, et al., "Swarmanoid: a novel concept for the study of heterogeneous robotic swarms," Robotics and Automation Magazine, IEEE, vol/issue: 20(4), pp. 60-71, 2013.

[33] A. V. Savkin, et al., "Distributed formation building algorithms for groups of wheeled mobile robots," Robotics and Autonomous Systems, vol. 75, pp. 463-474, 2016.

[34] T. S. Levitt and D. T. Lawton, "Qualitative navigation for mobile robots," Artificial intelligence, vol/issue: 44(3), pp. 305-360, 1990.

[35] J. A. Meyer and D. Filliat, "Map-based navigation in mobile robots: a review of map-learning and path-planning strategies," Cognitive Systems Research, vol/issue: 4(4), pp. 283-317, 2003.

[36] H. D. Whyte and T. Bailey, "Simultaneous localization and mapping: part I," Robotics and Automation Magazine, IEEE, vol/issue: 13(2), pp. 99-110, 2006.

[37] B. Tovar, et al., "Planning exploration strategies for simultaneous localization and mapping," Robotics and Autonomous Systems, vol/issue: 54(4), pp. 314-331, 2006.

[38] J. Clemens, et al., "An evidential approach to SLAM, path planning, and active exploration," International Journal of Approximate Reasoning, vol. 73, pp. 1-26, 2016.

[39] S. Nurmaini and S. Z. M. Hashim, "Motion planning in unknown environment using an interval fuzzy type-2 and neural network classifier," in Computational Intelligence for Measurement Systems and Applications, IEEE International Conference on, pp. 50-55, 2009.

[40] A. J. Sharkey and N. Sharkey, "The application of swarm intelligence to collective robots," Advances in applied artificial intelligence, pp. 157, 2006.

[41] G. Beni, "From swarm intelligence to swarm robotics," in Swarm robotics, Springer Berlin Heidelberg, pp. 1-9, 2004.

[42] F. Mondada, et al., "SWARM-BOT: A new distributed robotic concept," Autonomous Robots, vol/issue: 17(2-3), pp. 193-221, 2004.

[43] E. Trulls, et al., "Autonomous navigation for mobile service robots in urban pedestrian environments," Journal of Field Robotics, vol/issue: 28(3), pp. 329-354, 2011.

[44] K. J. O'Hara, et al., "Physical path planning using a pervasive embedded network," IEEE Transactions on Robotics, vol/issue: 24(3), pp. 741-746, 2008.

[45] P. Miller, "The smart swarm: How understanding flocks, schools, and colonies can make us better at communicating, decision making, and getting things done," Avery Publishing Group, Inc., 2010.

[46] N. Palmieri and S. Marano, "Discrete Firefly Algorithm for Recruiting Task in a Swarm of Robots," in NatureInspired Computation in Engineering, Springer International Publishing, pp. 133-150, 2016.

[47] R. Poli, "Analysis of the publications on the applications of particle swarm optimisation," Journal of Artificial Evolution and Applications, vol. 3, 2008.

[48] G. Lee and N. Y. Chong, "Flocking controls for swarms of mobile robots inspired by fish schools," INTECH Open Access Publisher, 2008. 
[49] V. Trianni, et al., "Evolving aggregation behaviors in a swarm of robots," in Advances in artificial life, Springer Berlin Heidelberg, pp. 865-874, 2003.

[50] S. Doctor, et al., "Optimal PSO for collective robotic search applications," in Evolutionary Computation, CEC2004. Congress on, vol. 2, pp. 1390-1395, 2004.

[51] L. S. Marcolino and L. Chaimowicz, "No robot left behind: Coordination to overcome local minima in swarm navigation," in Robotics and Automation, ICRA 2008. IEEE International Conference on, pp. 1904-1909, 2008.

[52] M. S. Couceiro, et al., "A novel multi-robot exploration approach based on particle swarm optimization algorithms," in Safety, Security, and Rescue Robotics, 2011 IEEE International Symposium on, pp. 327-332, 2011.

[53] O. Trullier, et al., "Biologically based artificial navigation systems: Review and prospects," Progress in neurobiology, vol/issue: 51(5), pp. 483-544, 1997.

[54] S. Thrun, "Probabilistic algorithms in robotics," Ai Magazine, vol/issue: 21(4), pp. 93, 2000.

[55] J. A. Meyer and D. Filliat, "Map-based navigation in mobile robots:: Ii. a review of map-learning and path-planning strategies," Cognitive Systems Research, vol/issue: 4(4), pp. 283-317, 2003.

[56] Koren and Borenstein, "Potential field methods and their inherent limitations for mobile robot navigation," in Robotics and Automation, Proceedings., 1991 IEEE International Conference on, pp. 1398-1404, 1991.

[57] S. X. Yang, et al., "A layered goal-oriented fuzzy motion planning strategy for mobile robot navigation," IEEE transactions on systems, man, and cybernetics - part b: cybernetics, vol/issue: 35(6), pp. 1214-1224, 2005.

[58] K. M. Krishna and P. K. Kalra, "Perception and remembrance of the environment during real-time navigation of a mobile robot," Robot. Automation System, vol. 37, pp. 25-51, 2001

[59] Y. H. Liu and S. Arimoto, "Path planning using a tangent graph for mobile robots among polynomial and curved obstacles," The International Journal of Robotics Research, vol/issue: 11(4), pp. 376-82, 1992.

[60] Kant K. and Zucker S. W., "Towards efficient planning: the path velocity decomposition," The International Journal of Robotics Research, vol/issue: 5(1), pp. 72-89, 1986.

[61] K. Fujimura and H. Samet, “Accessibility: a new approach to path planning among moving obstacles," Proceedings of IEEE conference on computer vision and pattern recognition, Ann Arbor, MI, pp. 803-810, 1988.

[62] J. G. Lamadrid and M. L. Gini, "Path tracking through uncharted moving obstacles," International Journal IEEE Transactions on Systems, Man and Cybernetics, vol/issue: 20(6), pp. 1408-1422, 1990.

[63] M. G. Slack and D. P. Miller, "Path planning through time and space in dynamic domains," Proceedings of the international joint conference on artificial intelligence, pp. 1067-1070, 1987.

[64] P. Fiorini and Z. Shiller, "Motion planning in dynamic environments using the relative velocity paradigm," Proceedings of IEEE conference on robotics and automation, pp. 560-565, 1993.

[65] R. Simmons, "The curvature-velocity method for local obstacle avoidance," Proceedings of the international conference on robotics and automation, vol. 4, pp. 2275-2282, 1996.

[66] H. Hu, et al., "A modular architecture for autonomous robots," Microprocessor and Microsystems, vol. 21, pp. 349361,1998

[67] E. Tunstel and M. Jamshidi, "Embedded Fuzzy Logic based Wall-following Behavior for Mobile Robot Navigation," Proceeding of Fuzzy Information Processing Society, pp. 329 - 330, 1994.

[68] S. Thrun, "Robotic mapping: A survey," Exploring artificial intelligence in the new millennium, vol. 1, pp. 1-35, 2002.

[69] B. N. Hui and K. D. Pratihar, "A comparative study on some navigation schemes of a real robot tackling moving obstacles," Robotics and Computer Integrated Manufacturing, vol. 25, pp. 810-828, 2009.

[70] S. X. Yang, et al., "An embedded fuzzy controller for a behavior-based mobile robot with guaranteed performance," IEEE Transactions on Fuzzy Systems, vol/issue: 12(4), pp. 436-446, 2004.

[71] L. A. Zadeh, "Fuzzy logic, neural networks, and soft computing," Communications of the ACM, vol/issue: 37(3), pp. 77-85, 1994.

[72] A. Zhu and X. S. Yang, "A fuzzy logic approach to reactive navigation of behavior-based mobile robots," Proceedings International Conference on Robotics and Automation, New-Orleans, pp. 5045-5050, 23-33, 2004.

[73] S. Jin and B. J. Choi, "Fuzzy logic system based obstacle avoidance for a mobile robot control and automation, and energy system engineering," Springer, pp. 1-6, 2011.

[74] P. Melin, et al., "Optimal design of type-2 and type-1 fuzzy tracking controllers for autonomous mobile robots under perturbed torques using a new chemical optimization paradigm," Expert Systems with Applications, vol/issue: 40(8), pp. 3185-3195, 2013.

[75] K. Samsudin, et al., "A highly interpretable fuzzy rule base using ordinal structure for obstacle avoidance of mobile robot," Applied Soft Computing, vol/issue: 11(2), pp. 1631-1637, 2011.

[76] J. M. Mendel, "Fuzzy sets for words: a new beginning," Proceeding of IEEE International Conf. on Fuzzy Systems, St. Louis, pp. 37-42, 2003.

[77] J. M. Mendel, "Uncertainty, fuzzy logic, and signal processing," Signal Proc. J., vol. 80, pp. 913-933, 2000.

[78] G. J. Klir and T. A. Folger, "Fuzzy sets, uncertainty, and information," Prentice-Hall, 1988.

[79] C. Wagner and H. Hagras, "Evolving type-2 fuzzy logic controllers for autonomous mobile robots," Analysis and design of intelligent systems using soft computing techniques, pp. 16-25, 2007.

[80] C. J. Kim and D. Chwa, "Obstacle avoidance method for wheeled mobile robots using interval type-2 fuzzy neural network," IEEE Transactions on Fuzzy Systems, vol/issue: 23(3), pp. 677-687, 2015.

[81] E. Kayacan, et al., "Towards agrobots: trajectory control of an autonomous tractor using type-2 fuzzy logic controllers," Mechatronics, IEEE/ASME Transactions on, vol/issue: 20(1), pp. 287-298, 2015.

[82] $\mathrm{H}$. Wu and J. Mendel, "Uncertainty bounds and their use in the design of interval type-2 fuzzy logic systems," IEEE Transaction on Fuzzy Systems, vol. 10, pp. 622-639, 2002. 
[83] H. Hagras, "Type-2 FLCs: a new generation of fuzzy controllers," IEEE Computational Intelligence Magazine, vol/issue: 2(1), pp. 30-43, 2007.

[84] R. Martínez, et al., "Optimization of interval type-2 fuzzy logic controllers for a perturbed autonomous wheeled mobile robot using genetic algorithms," Soft Computing for Hybrid Intelligent Systems. Springer Berlin Heidelberg, pp. 3-18, 2008.

[85] M. Biglarbegian, et al., "Design of novel interval type-2 fuzzy controllers for modular and reconfigurable robots: theory and experiments," IEEE Transactions on Industrial Electronics, vol. 99, 2010.

[86] Q. Liang and J. M. Mendel, "Interval type-2 fuzzy logic systems: theory and design," IEEE Transactions on Fuzzy systems, vol/issue: 8(5), pp. 535-550, 2000.

[87] A. Zhu and X. S. Yang, "Neurofuzzy-based approach to mobile robot navigation in unknown environments," IEEE Transaction on Systems, Man and Cybernetics-Part C: Applications and Reviews, vol/issue: 37(4), pp. 610-621, 2007.

[88] X. Wang, et al., "Mobile robots' modular navigation controller using spiking neural networks," Neurocomputing, vol. 134, pp. 230-238, 2014.

[89] Y. Quinonez, et al., "Autonomous Robot Navigation Based on Pattern Recognition Techniques and Artificial Neural Networks," in Bioinspired Computation in Artificial Systems, Springer International Publishing, pp. 320329, 2015.

[90] S. X. Yang and M. Meng, "An efficient neural network method for real-time motion planning with safety consideration," Robot. Auton. Syst., vol. 32, pp. 115-128, 2000.

[91] L. Moreno, et al., "A genetic algorithm for mobile robot localization using ultrasonic sensors," Journal of Intelligent and Robotic Systems, vol/issue: 34(2), pp. 135-154, 2002.

[92] K. H. Sedighi, et al., "Autonomous local path planning for a mobile robot using a genetic algorithm," in Evolutionary Computation, Congress on, vol. 2, pp. 1338-1345, 2004.

[93] W. Manikas, et al., "Genetic algorithms for autonomous robot navigation," Instrumentation and Measurement Magazine, IEEE, vol/issue: 10(6), pp. 26-31, 2007.

[94] A. T. Ismail, et al., "A mobile robot path planning using genetic algorithm in static environment," Journal of Computer Science, vol/issue: 4(4), pp. 341-344, 2008.

[95] A. P. A. Castro and J. D. S. De Silva, "Restoring images with a multi-scale neural network based technique," IEEE World Congress on Computational Intelligence, Hongkong, pp. 3197-3203, 2008.

[96] J. M. Mendel, "Uncertain rule-based fuzzy logic systems: Introduction and new directions," Upper Saddle River, NJ: Prentice-Hall, 2001.

[97] D. Goldberg, "Genetic Algorithms in Search, Optimization and Machine Learning," Norwell, MA: Kluwer, 1989.

[98] J. Kennedy and R. C. Eberhart, "Particle swarm optimization," Proc. IEEE int'l conf. on neural networks, vol. IV, pp. 1942-1948, 1995.

[99] J. Pugh and A. Martinoli, "Inspiring and modeling multi-robot search with particle swarm optimization," in Swarm Intelligence Symposium, pp. 332-339, 2007.

[100] Y. Zhang, et al., "Robot path planning in uncertain environment using multi-objective particle swarm optimization," Neurocomputing, vol. 103, pp. 172-185, 2013.

[101] H. Mo and L. Xu, "Research of biogeography particle swarm optimization for robot path planning," Neurocomputing, vol. 148, pp. 91-99, 2015.

[102] R. Zou, et al., "Particle swarm optimization-based source seeking," Automation Science and Engineering, IEEE Transactions on, vol/issue: 12(3), pp. 865-875, 2015.

[103] A. Banks, et al., "A review of particle swarm optimization. Part I: background and development," Natural Computing, vol/issue: 6(4), pp. 467-484, 2007.

[104] R. C. Eberhart and Y. Shi, "Particle swarm optimization: developments, applications and resources," in Evolutionary Computation, 2001. Proceedings of the 2001 Congress on, vol. 1, pp. 81-86, 2001.

[105] M. Dorigo and T. Stützle, "The ant colony optimization metaheuristic: Algorithms, applications, and advances," in Handbook of metaheuristics, pp. 250-285, 2003.

[106] B. C. Mohan and R. Baskaran, "A survey: Ant Colony Optimization based recent research and implementation on several engineering domain," Expert Systems with Applications, vol/issue: 39(4), pp. 4618-4627, 2012.

[107] D. Karaboga and B. Basturk, "Artificial Bee Colony (ABC) Optimization Algorithm for Solving Constrained Optimization Problems, IFSA, LNCS: Advances in Soft Computing," Foundations of Fuzzy Logic and Soft Computing, vol. 4529, pp. 789-798, 2007.

[108] X. Yang and X. He, "Firefly algorithm: recent advances and applications," Int. J. Swarm Intell., vol/issue: 1(1), pp. $36-50,2013$.

[109] X. Hu, et al., "Engineering optimization with particle swarm," in Swarm Intelligence Symposium, 2003. SIS'03. Proceedings of the 2003 IEEE, pp. 53-57, 2003.

[110] H. Wang, et al., "Opposition-based particle swarm algorithm with Cauchy mutation," in Evolutionary Computation, 2007. CEC 2007. IEEE Congress on, pp. 4750-4756, 2007.

[111] S. Kiranyaz, et al., "Fractional particle swarm optimization in multidimensional search space," Systems, Man, and Cybernetics, Part B: Cybernetics, IEEE Transactions on, vol/issue: 40(2), pp. 298-319, 2010.

[112] E. Bonabeau, et al., "Swarm intelligence: from natural to artificial systems (No. 1)," Oxford university press, 1999.

[113] M. Dorigo, et al., "Ant colony optimization," Computational Intelligence Magazine, IEEE, vol/issue: 1(4), pp. 2839, 2006. 
[114] M. Dorigo and T. Stützle, "Ant colony optimization: overview and recent advances," Techreport, IRIDIA, Universite Libre de Bruxelles, 2009.

[115] Q. H. Meng, et al., "Multi-robot odor-plume tracing in indoor natural airflow environments using an improved ACO algorithm," in Robotics and Biomimetics (ROBIO), IEEE International Conference on, pp. 110-115, 2010.

[116] M. Turduev, et al., "Experimental studies on chemical concentration map building by a multi-robot system using bio-inspired algorithms," Autonomous agents and multi-agent systems, vol/issue: 28(1), pp. 72-100, 2014.

[117] N. R. Hoff, et al., "Two foraging algorithms for robot swarms using only local communication," in Robotics and Biomimetics (ROBIO), 2010 IEEE International Conference on, pp. 123-130, 2010.

[118] O. Zedadra, et al., "A Cooperative Switching Algorithm for Multi-Agent Foraging," Engineering Applications of Artificial Intelligence, vol. 50, pp. 302-319, 2016.

[119] J. P. Hecker and M. E. Moses, "Beyond pheromones: evolving error-tolerant, flexible, and scalable ant-inspired robot swarms," Swarm Intelligence, vol/issue: 9(1), pp. 43-70, 2015.

[120] J. H. Liang and C. H. Lee, "Efficient collision-free path-planning of multiple mobile robots system using efficient artificial bee colony algorithm," Advances in Engineering Software, vol. 79, pp. 47-56, 2015.

[121] M. A. Contreras-Cruz, et al., "Mobile robot path planning using artificial bee colony and evolutionary programming," Applied Soft Computing, vol. 30, pp. 319-328, 2015.

[122] Y. Zhou, et al., "A novel path planning algorithm based on plant growth mechanism," Soft Computing, pp. 1-11, 2016.

[123] X. S. Yang, "Analysis of firefly algorithms and automatic parameter tuning," Emerging Research on Swarm Intelligence and Algorithm Optimization, vol. 36, 2014.

[124] S. K. Pal, et al., "Comparative study of firefly algorithm and particle swarm optimization for noisy non-linear optimization problems," International Journal of Intelligent Systems and Applications, vol/issue: 4(10), pp. 50, 2012.

[125] A. Baykasoglu, et al., "Artificial bee colony algorithm and its application to generalized assignment problem," Swarm Intelligence: Focus on Ant and particle swarm optimization, pp. 113-144, 2007.

[126] J. H. Lin, "Synergistic Strategies for Meta-heuristic Optimization Lerning Algorithms," International Journal of Engineering and Technical Research (IJETR), vol/issue: 4(4), 2016.

[127] G. Wang, et al., "A modified firefly algorithm for UCAV path planning," International Journal of Hybrid Information Technology, vol/issue: 5(3), pp. 123-144, 2012.

[128] C. Liu, et al., "A new path planning method based on firefly algorithm," in Computational Sciences and Optimization (CSO), 2012 Fifth International Joint Conference on, pp. 775-778, 2012.

[129] A. H. Paniagua, et al., "Solving the multi-objective path planning problem in mobile robotics with a firefly-based approach," Soft Computing, pp. 1-16, 2015.

[130] F. Mondada, et al., "The cooperation of swarm-bots: Physical interactions in collective robotics," Robotics and Automation Magazine, IEEE, vol/issue: 12(2), pp. 21-28, 2005.

[131] A. G. Pires, et al., "Towards Cooperative Localization in Robotic Swarms," in Distributed Autonomous Robotic Systems, pp. 105-118, 2016.

[132] D. Jeong and K. Lee, "Distributed Communication and Localization Algorithms for Homogeneous Robotic Swarm," in Distributed Autonomous Robotic Systems, pp. 405-418, 2016.

[133] J. E. Guivant, et al., "Simultaneous localization and map building using natural features and absolute information," Robotics and Autonomous Systems, vol/issue: 40(2), pp. 79-90, 2002.

[134] G. Jose, et al., "Simultaneous localization and map building using natural features in outdoor environments," Intelligent Autonomous Systems, vol/issue: 6(1), 2000.

[135] Brock O. and Khatib O., "High-speed navigation using the global dynamic window approach," Proceedings of IEEE International Conference on Robotics and Automation, Detroit, USA, pp. 341- 346, 1999.

[136] A. Saffiotti, "The uses of fuzzy logic in autonomous robot navigation," Journal Soft Computing, vol/issue: 1(4), pp. 180-197, 1997.

[137] A. Leslie, et al., "Intelligent Control of an Autonomous Mobile Robot using Type-2 Fuzzy Logic," Engineering Letters, vol/issue: 13(2), pp. 93-97, 2006.

[138] C. Wagner and H. Hagras, "Evolving type-2 fuzzy logic controllers for autonomous mobile robots," Analysis and design of intelligent systems using soft computing techniques. Springer Berlin Heidelberg, pp. 16-25, 2007.

[139] X. Wang, et al., "Mobile robots' modular navigation controller using spiking neural networks," Neurocomputing, vol. 134, pp. 230-238, 2014.

[140] I. Pratihar, et al., "Fuzzy-genetic algorithms and mobile robot navigation among static obstacles," Evolutionary Computation, CEC 99. Proceedings of the Congress on., vol. 1, 1999.

[141] M. J. Phinni, et al., "Obstacle Avoidance of a Wheeled Mobile Robot: A Genetic-NeuroFuzzy Approach," IISc Centenary - International Conference on Advances in Mechanical Engineering, India, 2008.

[142] P. Rusu, et al., "Behavior-Based Neuro-Fuzzy Controller for Mobile Robot Navigation," IEEE Transactions on Instrumentation and Measurement, vol/issue: 52(4), pp. 1335-1340, 2003.

[143] V. Ganapathy, et al., "Fuzzy and Neural Controllers for Acute Obstacle Avoidance in Mobile Robot Navigation," IEEE/ASME International Conference on Advanced Intelligent Mechatronics, Suntec Convention and Exhibition Center, Singapore, pp.1236-1241, 2009.

[144] Y. Q. Qin, et al., "Path planning for mobile robot based on particle swarm optimization," Robot, vol/issue: 26(3), pp. 222-225, 2004. 
[145] W. Jatmiko, et al., "A pso-based mobile robot for odor source localization in dynamic advection-diffusion with obstacles environment: theory, simulation and measurement," IEEE Computational Intelligence Magazine, vol/issue: 2(2), pp. 37-51, 2007.

[146] A. Cherubini and F. Chaumette, "Visual navigation of a mobile robot with laser-based collision avoidance," The International Journal of Robotics Research, vol/issue: 32(2), pp. 189-205, 2013.

[147] C. H. Hsu and C. F. Juang, "Evolutionary robot wall-following control using type-2 fuzzy controller with speciesDE-activated continuous ACO,” IEEE Transactions on Fuzzy Systems, vol/issue: 21(1), pp. 100-112, 2013.

[148] A. Elfes, "Sonar-based real-world mapping and navigation," IEEE Journal on Robotics and Automation, vol/issue: 3(3), pp. 249-265, 1987.

[149] E. Gat, "Integrating planning and reacting in a heterogeneous asynchronous architecture for controlling real-world mobile robots," in $A A A i$, vol. 1992, pp. 809-815, 1992.

[150] E. M. Albor, et al., "Mobile Robot with Movement Detection Controlled by a Real-Time Optical Flow Hermite Transform," in Nature-Inspired Computing for Control Systems, pp. 231-263, 2016.

[151] J. M. Mendel and R. B. John, "Type-2 fuzzy sets made simple," IEEE Transactions on fuzzy systems, vol/issue: 10(2), pp. 117-127, 2002.

[152] S. Thompson, et al., "A probabilistic model of human motion and navigation intent for mobile robot path planning," in Autonomous Robots and Agents, 2009. ICARA 2009. 4th International Conference on, pp. 663-668, 2009.

[153] S. Vechet, et al., "Enhancement of Autonomous Robot Navigation via Sensor Failure Detection," Advanced Mechatronics Solutions. Springer, Cham, pp. 657-662, 2016.

[154] H. Wang, et al., "Opposition-based particle swarm algorithm with Cauchy mutation," in Evolutionary Computation, 2007. CEC 2007. IEEE Congress on, pp. 4750-4756, 2007.

[155] Y. Koren and J. Borenstein, "Potential field methods and their inherent limitations for mobile robot navigation," in Robotics and Automation, Proceedings, 1991 IEEE International Conference on. pp. 1398-1404, 1991. 